\title{
Cell-cycle dependent micronucleus formation and mitotic disturbances induced by 5 -azacytidine in mammalian cells
}

\author{
Helga Stopper ${ }^{\text {a }}$, Carsten Körber ${ }^{\text {a }}$, Dietmar Schiffmann ${ }^{\text {a }}$ and William J. Caspary ${ }^{\text {b }}$ \\ a Institute of Pharmacology and Toxicology, University of Würzburg, W-8700 Würburg, Germany and \\ ${ }^{b}$ Cancer and Molecular Genetics Group, National Institute of Environmental Health Sciences, National Institutes of Health, \\ Research Triangle Park, NC 27709, USA
}

(Received 25 August 1992)

(Revision received 16 December 1992)

(Accepted 8 February 1993)

Keywords: Micronuclei; L5178Y cells; 5-Azacytidine; Berenil; DES; Ethionine; Mitosis

\section{Summary}

5-Azacytidine was originally developed to treat human myelogenous leukemia. However, interest in this compound has expanded because of reports of its ability to affect cell differentiation and to alter eukaryotic gene expression. In an ongoing attempt to understand the biochemical effects of this compound, we examined the effects of 5-azacytidine on mitosis and on micronucleus formation in mammalian cells. In L5178Y mouse cells, 5-azacytidine induced micronuclei at concentrations at which we and others have already reported its mutagenicity at the $t k$ locus. Using CREST staining and C-banding studies, we showed that the induced micronuclei contained mostly chromosomal fragments although some may have contained whole chromosomes. By incorporating BrdU into the DNA of SHE cells, we determined that micronuclei were induced only when the compound was added while the cells were in S phase. Microscopically visible effects due to 5-azacytidine treatment were not observed until anaphase of the mitosis following treatment or thereafter. 5-Azacytidine did not induce micronuclei via interference with formation of the metaphase chromosome arrangement in mitosis, a common mechanism leading to aneuploidy. Supravital UV microscopy revealed that chromatid bridges were observed in anaphase and, in some cases, were sustained into interphase. In the first mitosis after 5-azacytidine treatment we observed that many cells were unable to perform anaphase separation. All of these observations indicate that 5 -azacytidine is predominantly a clastogen through its incorporation into DNA.

Correspondence: Dr. William J. Caspary, NIEHS, P.O. Box 12233, Research Triangle Park, NC 27709, USA.

Abbreviations: BrdU, bromodeoxyuridine; BSA, bovine serum albumin; DES, diethylstilbestrol; DMSO, dimethyl sulfoxide; FITC, fluorescein isothiocyanate; PBS, phosphate-buffered saline; SHE cells, Syrian hamster embryo cells; TFT, trifluorothymidine.
5-Azacytidine was originally developed to treat human myelogenous leukemia (Von Hoff et al., 1976). However, interest in this compound has expanded because of reports of its ability to affect cell differentiation and to alter eukaryotic gene expression (Holliday, 1989)

Most of 5-azacytidine's biological activity, including its ability to induce gene expression, has 
been attributed to its capability to induce DNA hypomethylation. Jones and coworkers demonstrated a correlation between the ability of a series of cytidine analogs, including 5-azacytidine, to induce differentiation and their ability to prevent DNA methylation (Constantinides et al., 1977; Jones and Taylor, 1980; Taylor and Jones, 1979). However, 5-azacytidine has also been shown to be mutagenic in a variety of cell systems (Amacher and Turner, 1987; Call et al., 1986; McGregor et al., 1989; Schmuck et al., 1988) and using molecular biological techniques, we have found that the compound induces point mutations in AS52 cells (Daston et al., 1992; Kelecsényi et al., 1993). We (McGregor et al., 1989) have also evaluated the same cytidine analogs evaluated by Jones and coworkers (Constantinides et al., 1977; Jones and Taylor, 1980; Taylor and Jones, 1979) for their ability to induce trifluorothymidine resistance (TFT ${ }^{r}$ ) in $\mathrm{L} 5178 \mathrm{Y}$ mouse cells. We found that their mutagenic potency correlated with their reported ability to induce differentiation in C3H10T1/2 cells (Jones and Taylor, 1980). Thus, it has been reported that both the mutagenic and hypomethylating ability of these azacytidine analogs correlated with their ability to induce differentiation of cells in culture.

5-Azacytidine has also been shown to affect chromosome structure. We have reported that this compound induces micronuclei in SHE cells (Stopper et al., 1992). Effects such as chromosome decondensation, endoreduplication and segmentation have also been reported (Hori, 1983; Parrow et al., 1989; Schmid et al., 1984). Major perturbations of cell cycle kinetics together with elevated rates of endomitosis and tetraploidy were found in human fibroblast-like cells from skin and amniotic fluid (Poot et al., 1990). This is consistent with the findings of Zatsepina et al. (1989) who showed that 5-azacytidine blocked pig kidney cells in $G_{2}$ phase of the cell cycle.

5-Azacytidine is clastogenic. Shelby and coworkers have evidence that 5-azacytidine treatment of $\mathrm{CHO}$ cells produced a dose dependent increase in chromosome aberrations in these cells in culture (Shelby, 1992). The compound was also found to be clastogenic in plant cells (Fucik et al., 1970) and human lymphoblasts from a male donor (Lavia et al., 1985). Call et al. (1986) failed to show an increase in chromosomal aberrations in human lymphoblasts in culture and Van Hummelen and coworkers asserted that the compound is an aneugen (Van Hummelen et al., 1992) in mouse bone-marrow cells in vivo.

5-Azacytidine induces micronuclei (Stopper et al., 1992). Micronuclei can contain chromosomal fragments or whole chromosomes. The contents of micronuclei depend on the mechanisms leading to their formation. The critical lesion in the formation of chromosomal aberrations is presumed to be double-strand breaks while aneuploidy is thought to be induced by disturbances in chromosome distribution during mitosis.

The purpose of the present work is to characterize the contents of 5-azacytidine-induced micronuclei and to examine the mitotic disturbances that may be involved in micronucleus induction by 5-azacytidine treatment. In addition to SHE cells which we have previously used (Stopper et al., 1992), we chose L5178Y mouse cells because of their low incidence of spontaneous micronuclei (Kirchner et al., 1992) and because we have previously shown that 5-azacytidine induces TFT resistance in these cells (McGregor et al., 1989). In this paper we report that approximately $80 \%$ of the 5-azacytidine induced micronuclei did not stain for kinetochores and for centromeric heterochromatic DNA suggesting that 5-azacytidine is primarily a clastogen. However, a small percentage of the micronuclei could contain whole chromosomes. We, then, examined 5-azacytidinetreated cells for the mitotic disturbances that could lead to an aneugenic response.

\section{Materials and methods}

Chemicals. 5-Azacytidine ( $\mathrm{MW}=244)$, berenil $(M W=515.5)$, ethionine ( $M W=163.2)$, DES $(M W=268.4)$, bisbenzimid 33258 and 33342, BrdU and anti-mouse IgG FITC-conjugated antibody, anti- $\alpha$-tubulin antibody and FITC-conjugated goat anti-human antibody were purchased from Sigma Chemical Co., St. Louis, MO, USA. DMSO was acquired from Aldrich Co., Nettetal, Germany and CREST-serum (antikinetochore antibody) was purchased from Antibodies Inc, Davis, CA, USA. Anti-BrdU fluores- 
cein (monoclonal antibody against BrdU) was acquired from Boehringer, Mannheim, Germany.

Cell culture. L5178Y $t k^{+/-}$mouse lymphoma cells, clone 3.7.2c (Clive et al., 1972) were routinely cultured in suspension in RPMI-1640 supplemented with 95 units $/ \mathrm{ml}$ penicillin, $95 \mu \mathrm{g} / \mathrm{ml}$ streptomycin, $0.25 \mathrm{mg} / \mathrm{ml}$ L-glutamine, $107 \mu \mathrm{g} / \mathrm{ml}$ sodium pyruvate and $10 \%$ heat-inactivated horse serum (Boehringer Mannheim, Germany). Cell cultures were grown in humidified atmosphere with $5 \% \mathrm{CO}_{2}$ in air at $37^{\circ} \mathrm{C}$. All experiments with SHE cells were performed with tertiary or quaternary cultures derived from 13-day old Syrian hamster embryos. The culture medium used was IBR-modified Dulbecco's reinforced medium (Grand Island Biological Co.) supplemented with $100 \mathrm{U} / \mathrm{ml}$ penicillin and $100 \mu \mathrm{g} / \mathrm{ml}$ streptomycin, $3.7 \mathrm{~g} / 1 \mathrm{NaHCO}_{3}$ and $15 \%$ fetal calf serum (Gibco, Karlsruhe, Germany). Cell cultures were grown in humidified atmosphere with $12 \% \mathrm{CO}_{2}$ in air at $37^{\circ} \mathrm{C}$.

Micronucleus assay. Exponentially growing L5178Y mouse cells were treated with chemicals for $4 \mathrm{~h}$. If chemicals were dissolved in DMSO, the final concentration of DMSO did not exceed $1 \%$. After removing the chemicals by medium replacement, the cells were incubated for $5-20 \mathrm{~h}$ as specified in the table legends. At the indicated times the cells were deposited onto glass slides by cytospin centrifugation. Fixation was performed with methanol $\left(-20^{\circ} \mathrm{C}, 1 \mathrm{~h}\right)$. For staining, the slides were washed with distilled water, incubated with bisbenzimid $33258(1 \mu \mathrm{g} / \mathrm{ml}, 5 \mathrm{~min})$, washed 3 times with distilled water and mounted for fluorescence microscopy. Using a magnification or $1250 \times$, we scored the number of nuclei and micronuclei ( 3 slides with 2000 nuclei scored per slide).

Kinetochore staining. Kinetochore staining was achieved by incubating the fixed cell preparations (after rinsing with PBS) with CREST serum $(60 \mathrm{~min})$ in a humidified chamber at $37^{\circ} \mathrm{C}$. After rinsing with PBS, the cells were incubated as before with FITC conjugated goat anti-human antibody (diluted $1: 100$ in PBS), rinsed again and counter stained with bisbenzimid $33258(1 \mu \mathrm{g} / \mathrm{ml}$, $5 \mathrm{~min}$ ).

C-Banding. L5178Y cells were fixed and stained according to the conventional technique used for karyotyping (Sumner, 1972). Micronuclei were scored for the presence of dark stained c-band regions using a magnification of $1250 \times$. (The only micronuclei counted were those in cells where dark staining heterochromatic regions in the nucleus were clearly visible.)

Analysis of metaphase ring arrangement. We treated L5178Y cells for $4 \mathrm{~h}$ with the chemical. The cells were fixed at times indicated in table 3 . Fixation and staining was performed as described for micronuclei above. Due to cytospin preparation, chromosomes arranged at metaphase plate are seen as a ring arrangement. Metaphases showing a ring arrangement were evaluated for chromosomes dislocated from the ring. Spindle staining was achieved by applying anti- $\alpha$-tubulin antibody (diluted 1:250 in PBS) and FITClabelled anti-mouse antibody (diluted 1:100 in PBS) according to the procedure given for kinetochore staining.

Supravital UV microscopy. Supravital UV microscopy was performed according to the method of Schiffmann and DeBoni (1991) using SHE cells. This method allows the observation of the spacial arrangement of chromatin elements throughout mitosis in living cells. The cells were incubated with culture medium containing 5azacytidine and bisbenzimid $33342(0.1 \mu \mathrm{g} / \mathrm{ml}$; staining of cellular DNA) for $4 \mathrm{~h}$. Then the medium was replaced by culture medium containing bisbenzimid but no 5-azacytidine. Observation of the cells using supravital UV microscopy was carried out immediately after addition of 5azacytidine and up to $30 \mathrm{~h}$ thereafter. Control cells were untreated except bisbenzimid staining. Due to the fact that cells are followed individually for a number of hours, experiments were limited to low cell numbers.

BrdU incorporation. SHE cells were plated in 35-mm Petri dishes containing glass coverslips and allowed to grow. Then, the cell culture 


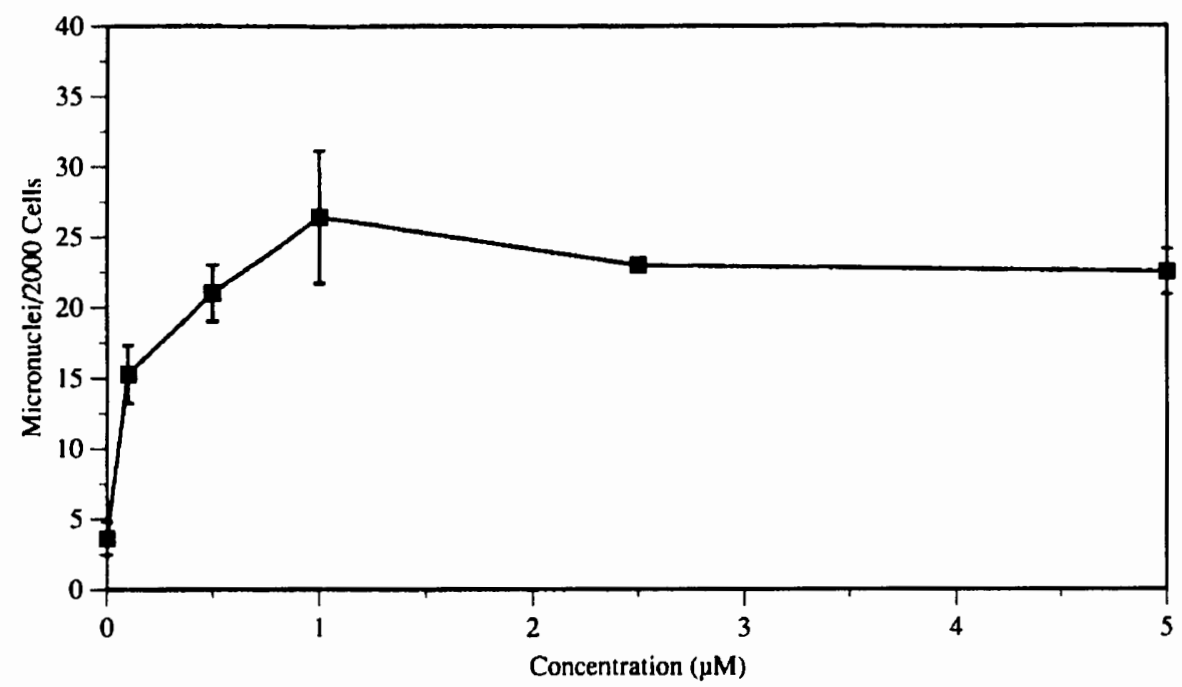

Fig. 1. Dose-response of 5-azacytidine induced micronuclei. L5178Y cells were treated with the indicated concentrations for $4 \mathrm{~h}$ and fixed $18 \mathrm{~h}$ later. Cells treated with $37 \mu \mathrm{M}$ DES showed $28.0 \pm 6.8$ micronuclei per 2000 cells.

medium was replaced by culture medium containing the chemical to be tested and $\operatorname{BrdU}(10 \mu \mathrm{M})$. Following an incubation period of $2.5 \mathrm{~h}$ the compound and $\mathrm{BrdU}$ were removed by changing the medium and washing the cells with culture medium once. After $12 \mathrm{~h}$, the cells were fixed with methanol for at least $60 \mathrm{~min}$ at $-20^{\circ} \mathrm{C}$. Coverslips were washed with PBS twice and treated with $\mathrm{HCl}(1.5 \mathrm{M})$. After $30 \mathrm{~min}$ they were washed twice within a 10-min period with
$\mathrm{Na}_{2} \mathrm{~B}_{4} \mathrm{O}_{7}(0.1 \mathrm{M}, \mathrm{pH} 8.5)$ for neutralization after the acid treatment. They were then washed twice with PBS within a 10 -min period. $20 \mu \mathrm{l}$ of antiBrdU fluorescein antibody $(50 \mu \mathrm{g} / \mathrm{ml}$ in PBS with $0.1 \%$ BSA) were dropped onto another slide and covered with the inverted coverslip. After incubation $\left(30-45 \mathrm{~min}, 37^{\circ} \mathrm{C}\right)$ in a humidified chamber, they were washed with PBS, counterstained with bisbenzimid $(1 \mu \mathrm{g} / \mathrm{ml}, 5 \mathrm{~min})$ and mounted for fluorescence microscopy. Nuclei and

\section{TABLE 1}

\section{KINETOCHORE STAINING OF MICRONUCLEI IN L5178Y CELLS}

\begin{tabular}{llll}
\hline Treatment & $\begin{array}{l}\text { Number } \mathrm{K}^{+} \mathrm{MN} \text { per } \\
100 \mathrm{MN} \text { examined }\end{array}$ & $\begin{array}{l}\text { Number of induced } \\
\mathrm{K}^{+} \mathrm{MN}\end{array}$ & $\begin{array}{l}\text { \% induced } \mathrm{MN} \\
\text { that are } \mathrm{K}^{+}\end{array}$ \\
\hline Vehicle control & 22 & - & - \\
5-Azacytidine $(1 \mu \mathrm{M})$ & $19.0 \pm 2.7$ & 16.0 & 18.6 \\
DES $(37 \mu \mathrm{M})$ & $77.3 \pm 4.2$ & 72.3 & 93.5 \\
Ethionine $(80 \mu \mathrm{M})$ & $28.0 \pm 3.6$ & 24.7 & 29.0 \\
Berenil $(155 \mu \mathrm{M})$ & $27.7 \pm 3.2$ & 26.6 & 28.0 \\
\hline
\end{tabular}

Cells were treated for $4 \mathrm{~h}$ and were fixed and stained after a recovery time of $20,20,5,20$ and $6 \mathrm{~h}$ for vehicle control, 5-azacytidine, DES, ethionine and berenil respectively. 3 slides were prepared from each treated culture and 100 micronuclei from each slide evaluated for the presence of kinetochores. Column 2 shows the percentage of micronuclei observed that stain positive for CREST antibody. From the dose-response curves we know that the percentage of induced (spontaneous) micronuclei in each population is 86.2 (13.8), $77.3(22.7), 85.1(14.9)$ and 95.0 (5) respectively. From this and the number of $\mathrm{K}^{+}$micronuclei in the vehicle control, we calculate the number of $\mathrm{K}^{+}$micronuclei that are induced by each chemical (column 3 ). The final column lists the percentage of chemically induced micronuclei that stain positive for the presence of kinetochore. $\mathrm{K}^{+}$means staining positive for the presence of kinetochores; MN, micronuclei. 
micronuclei were evaluated at $1250 \times$ magnification for the presence of BrdU antibody staining.

\section{Results}

Induction of micronuclei by 5-azacytidine. 5Azacytidine induced an increase in micronuclei at doses from 0.1 up to $5 \mu \mathrm{M}$, the highest dose we examined (Fig. 1). The micronucleus fraction increased to a value of 26.4 micronuclei per 2000 cells at $1 \mu \mathrm{M}$. At higher doses no further increase in the micronucleus fraction was observed. The spontaneous micronucleus fraction was 3.6 per 2000 cells. This dose-response curve is comparable to the micronucleus results obtained with SHE cells (Stopper et al., 1992).

To determine whether 5-azacytidine is a clastogen or an aneugen, we assayed the contents of the 5-azacytidine-induced micronuclei for the presence of kinetochores by immunofluorescent staining using CREST antibodies (Degrassi and Tanzarella, 1988). Our data (Table 1) show that $18.6 \%$ of the 5 -azacytidine-induced micronuclei stained positive in this assay indicating the absence of kinetochores in $81.4 \%$ of this micronucleus population. Therefore, the majority of 5 . azacytidine-induced micronuclei most likely did not contain whole chromosomes, but rather acentric chromosomal fragments. For comparison, 93.5\% of the micronuclei induced by DES, an agent known to induce aneuploidy in SHE cells
(Schiffmann and DeBoni, 1991), stained positive for kinetochores.

One criticism of the interpretation of CREST staining data is that whole chromosomes with damaged kinetochore protein may not stain positive in this assay. It is also possible that kinetochore binding to the chromosome may be disturbed and that such chromosomes may remain at the metaphase plate during mitosis. This would result in micronuclei with whole chromosomes that would not be detected by this technique.

To investigate this possibility, we performed two more experiments. We first stained the micronuclei with the c-banding technique commonly used for the staining of heterochromatic centromeric DNA regions in metaphase chromosomes. Our results (Table 2) show that a signal was present in $20 \%$ of the vehicle control micronuclei. Of the 5-azacytidine-induced micronuclei, $23.6 \%$ stained positive for the presence of centromeric heterochromatin. In contrast, $89.7 \%$ of the DES-induced micronuclei were positive in the staining procedure. We then evaluated the centromeric heterochromatin regions with bisbenzimid 33258 which preferentially stains these regions in mouse chromosomes. In this experiment, $20 \%$ of the micronuclei treated with vehicle control showed a signal ( 50 micronuclei evaluated). After correcting for the vehicle control, $27 \%$ of the 5 -azacytidine-induced cells $(100 \mathrm{mi}-$ cronuclei evaluated) showed a signal. A small

TABLE 2

CENTROMERE STAINING OF MICRONUCLEI IN LS178Y CELLS

\begin{tabular}{llll}
\hline Treatment & $\begin{array}{l}\text { Number } \mathrm{C}^{+} \mathrm{MN} \text { per } \\
100 \mathrm{MN} \text { examined }\end{array}$ & $\begin{array}{l}\text { Number of induced } \\
\mathrm{C}^{+} \mathrm{MN}\end{array}$ & $\begin{array}{l}\text { \% induced } \mathrm{MN} \\
\text { that are } \mathrm{C}^{+}\end{array}$ \\
\hline Vehicle control & 20 & - & - \\
5-Azacytidine $(1 \mu \mathrm{M})$ & $23.3 \pm 2.1$ & 20.5 & 23.6 \\
DES $(37 \mu \mathrm{M})$ & $74.3 \pm 7.6$ & 69.3 & 89.7 \\
\hline
\end{tabular}

Cells were treated for $4 \mathrm{~h}$ and fixed and stained after a recovery time of 20,20,5, vehicle control, 5-azacytidine and DES respectively. 3 slides were prepared from each treated culture and 100 micronuclei evaluated for the presence of centromeric DNA. Column 2 shows the fraction of micronuclei observed that stain positive for centromeres. From the dose-response curves we know that the percentage of induced (spontaneous) micronuclei in each population is 86.2 (13.8) and 77.3 (22.7) respectively. From this and the number of $\mathrm{C}^{+}$micronuclei in the vehicle control (20), we calculate the number of $\mathrm{C}^{+}$micronuclei that are induced by the chemical (column 3). The final column lists the percentage of chemically induced micronuclei that stain positive for the presence of centromeres. " $\mathrm{C}^{+}$" means staining positive for the presence of centromeres; $\mathrm{MN}$, micronuclei. 


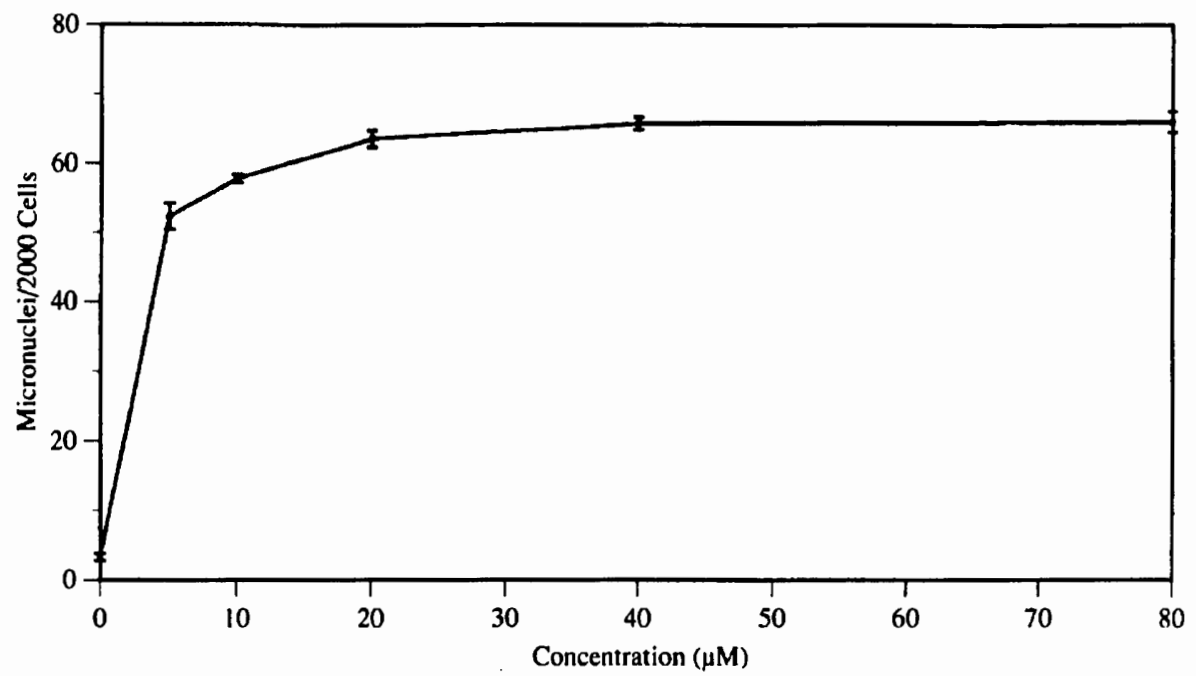

Fig. 2. Dose-response of ethionine induced micronuclei. L5178Y mouse cells were treated with the indicated concentrations of ethionine for $4 \mathrm{~h}$ and fixed $5 \mathrm{~h}$ later. The micronuclei fraction for the vehicle control was $3.3 \pm 0.5$ micronuclei per 2000 cells.

percentage of centromeres in micronuclei of 5azacytidine-treated cells may be without stainable kinetochore protein. However, the majority of micronuclei contain chromosomal fragments. Thus, we conclude that 5-azacytidine is predominantly a clastogen.

Treating cells with 5-azacytidine is known to result in hypomethylated DNA and many of the effects of this compound have been attributed to this property. Ethionine also prevents DNA methylation by inhibiting the enzymatically catalyzed formation of $S$-adenosylmethionine. This compound induced micronuclei in a dose dependent manner (Fig. 2). 5-Azacytidine has been reported to interfere with chromosome condensation (Fucik et al., 1970; Hori, 1983; Parrow et al., 1989; Schmid et al., 1984). Berenil, which causes chromosome undercondensation by binding to the minor groove of the DNA molecule, also induced micronuclei (Fig. 3). Of the micronuclei in both the ethionine and berenil treated cultures, 28\% stained positive in the CREST antibody assay for kinetochores (Table 1) suggesting fragments of chromosomes were present in most of these mi-

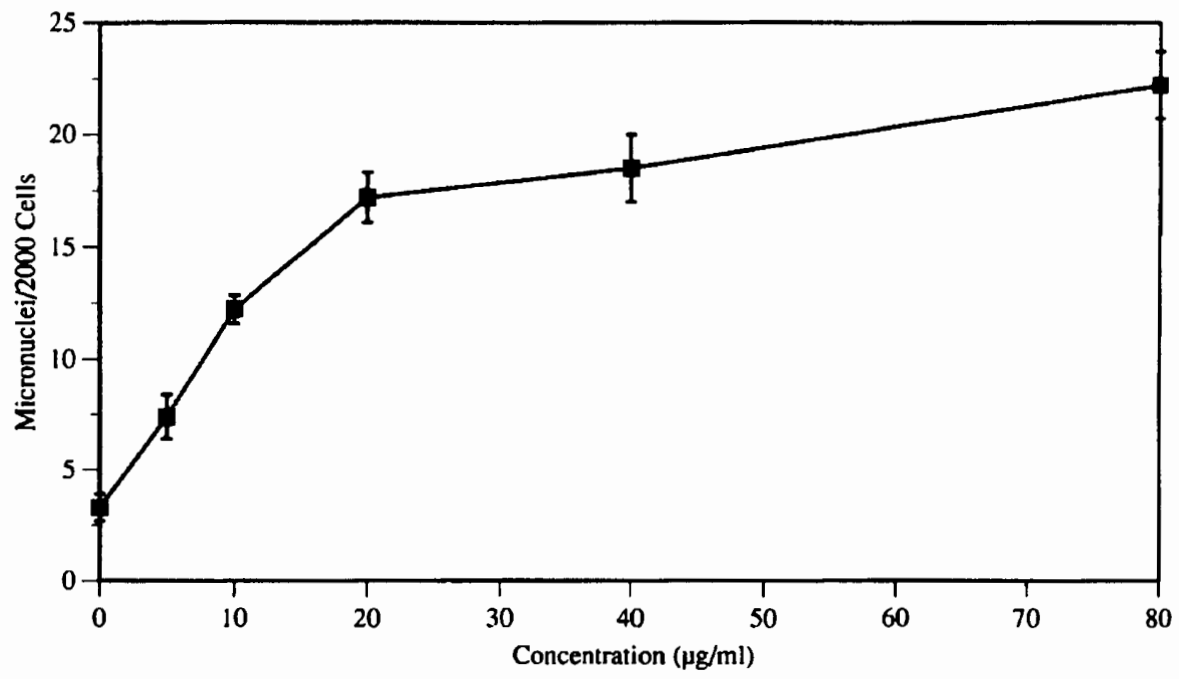

Fig. 3. Dose-response of berenil induced micronuclei. L5178Y mouse cells were treated with the indicated concentrations of berenil for $4 \mathrm{~h}$ and fixed $5 \mathrm{~h}$ later. The micronuclei fraction for the vehicle control was $3.3 \pm 0.6$ micronuclei per 2000 cells. 
cronuclei. The shapes of the dose-response curves and the percentages of kinetochores present for both these compounds are similar to that of 5-azacytidine.

To determine the stage of the cell cycle in which 5-azacytidine had to be added to induce micronuclei, we attempted to synchronize L5178Y and SHE cells. We tried fetal calf serum withdrawal, mitotic shake-off (SHE cells only), colcemid, aphidicolin and hydroxyurea. Some of the methods commonly used for cell synchronization induced micronuclei themselves and were not suitable for our purposes; others did not achieve synchronization of the cell types used. Therefore, we used a novel approach to study the effects of 5 -azacytidine in specific cell cycle stages. We treated SHE cells for $2.5 \mathrm{~h}$ with BrdU and 5azacytidine (or DES as a control) concurrently. After removing the chemicals, we fixed the cells $12 \mathrm{~h}$ later, a time that was short enough that cells would not pass through a second mitosis since the doubling time of SHE cells is $17 \mathrm{~h}$. We evaluated each cell and its associated micronucleus, if present, for the appearance of BrdU in the chromatin material using BrdU antibody staining. The presence of $\mathrm{BrdU}$ indicated that replication or repair had taken place during the time of the pulse treatment with 5-azacytidine (DES) and BrdU. We counted the number of cells with micronuclei in each of the following observed categories: (1) cells with $\mathrm{BrdU}$ signal in the nucleus and micronucleus; (2) cells with BrdU signal in the nucleus, but not in the micronucleus; and (3) $\mathrm{mi}$ cronucleus containing cells with no BrdU (Fig. 4).

We counted cells treated with the vehicle control in each of the categories (Fig. 4). Those that showed no BrdU in either the nuclei or micronuclei must have been in $G_{2}$ phase during treatment and formed micronuclei during the mitoses immediately following treatment or in $M$ phase, in which case the micronuclei would have been formed in the same mitosis in which treatment occurred. Cells exposed to the vehicle control in $\mathrm{G}_{1}$ that already had micronuclei would also appear in this category. However, those cells exposed in $G_{1}$ without micronuclei would not be able to form them because they would not have had time to undergo mitosis during the $12 \mathrm{~h}$ before fixation. Those vehicle control treated cells that incorporated $\mathrm{BrdU}$ into both the nucleus and micronucleus were in $\mathrm{S}$ phase during treatment (either with a previously existing micronu-

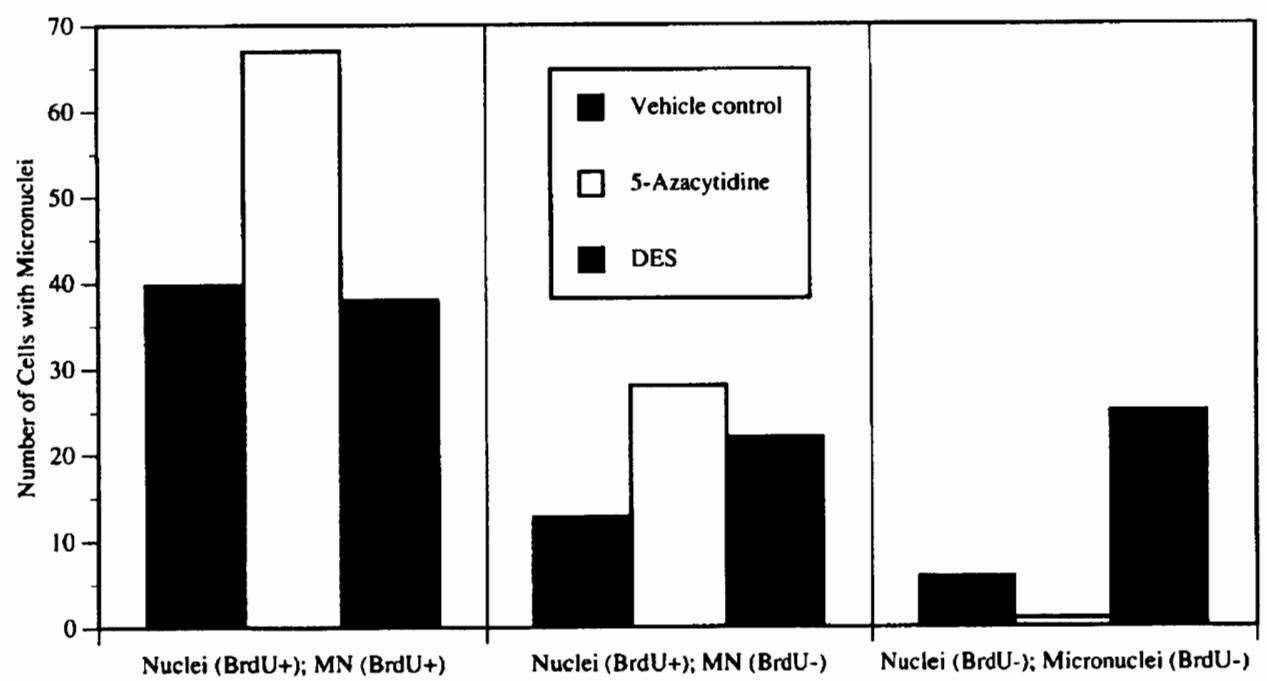

Fig. 4. Cell-cycle dependence of micronucleus induction. SHE cells were treated for $2.5 \mathrm{~h}$ with $1 \mu \mathrm{M} 5$-azacytidine or $47 \mu \mathrm{M}$ DES, each with $10 \mu \mathrm{M}$ BrdU. This graph shows the number of cells with micronuclei normalized to 2000 cells evaluated. The first category represents those cells that contain BrdU in both the nuclei and their associated micronuclei. The second category consists of those cells that possess BrdU in the nuclei but not in their associated micronuclei. In the last category are those cells that have not incorporated BrdU in either the nuclei or associated micronuclei. The number of cells evaluated were 10000 for the vehicle control, 8000 for 5-azacytidine-treated and 5000 for DES treated. 
TABLE 3

METAPHASE RING ARRANGEMENT IN L5178Y CELLS

\begin{tabular}{llll}
\hline Treatment & $\begin{array}{l}\text { Time } \\
\text { scored } \\
\text { (h) }\end{array}$ & $\begin{array}{l}\text { Number } \\
\text { mitotic } \\
\text { rings }\end{array}$ & $\begin{array}{l}\text { Number rings with } \\
\text { displaced } \\
\text { chromosomes }\end{array}$ \\
\hline $1 \mu \mathrm{M}$ & 0 & 300 & 1 \\
$5-$ Azacytidine & 1 & 300 & 0 \\
& 2.5 & 300 & 0 \\
$5 \mu \mathrm{M}$ & 5 & 335 & 0 \\
$5-$ Azacytidine & 0 & 300 & 0 \\
& 1 & 300 & 0 \\
37 $\mu$ M DES & 0 & 300 & 0 \\
Vehicle control & 0 & 300 & 0
\end{tabular}

Cells were treated with 5-azacytidine or DES for $4 \mathrm{~h}$ and fixed at the indicated times after that. Metaphase ring arrangements were analyzed for displaced chromosomes.

cleus or forming one in the next mitosis) or in a non-replicative phase of the cell cycle and undergoing repair.

5-Azacytidine treatment resulted in an increased number of cells with BrdU incorporation into the DNA of the nucleus and micronucleus compared to the control. DES-induced no in. crease in this category. Both compounds in-
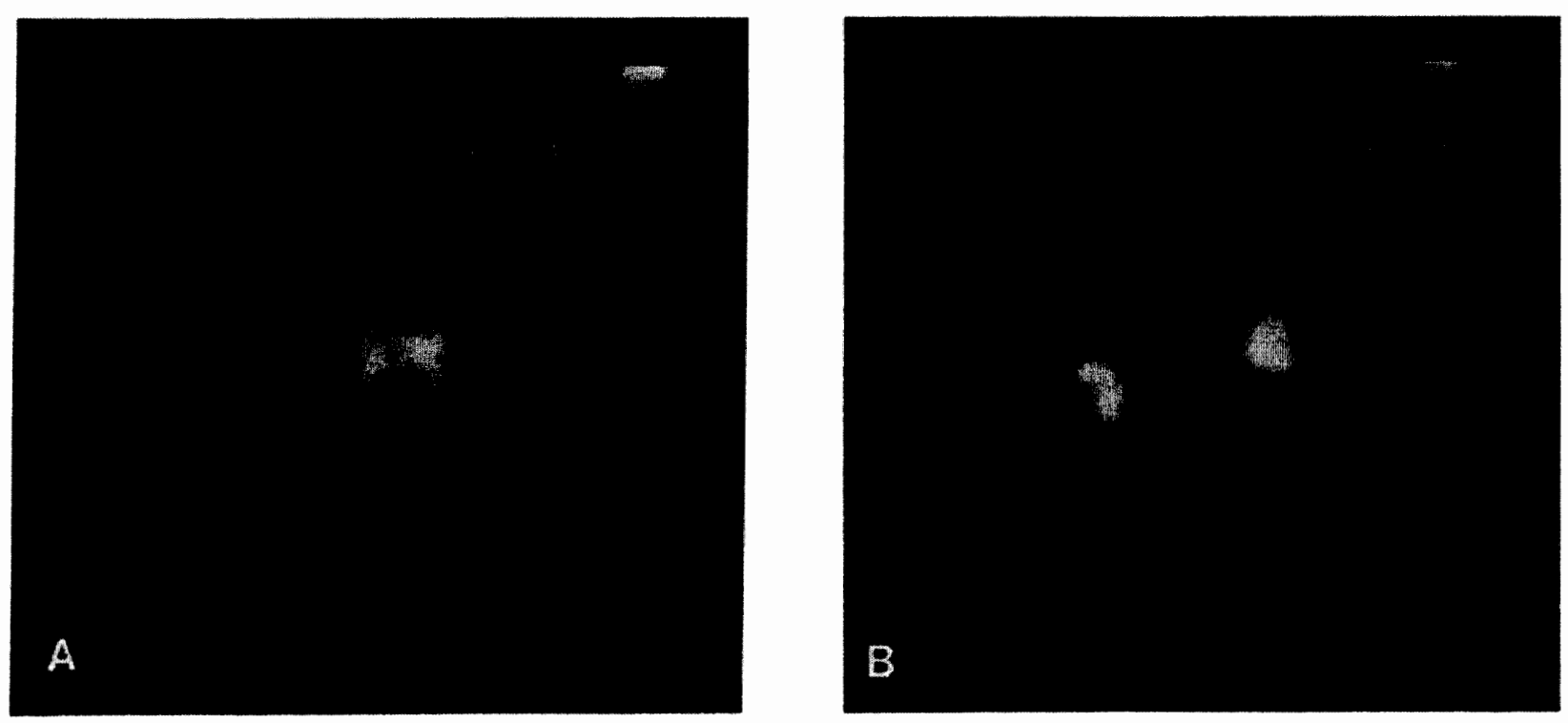

Fig. 5. Supravital UV microscopy observation of live SHE cells during the course of mitosis. Chromatin material is visible due to

creased the number of cells containing $\mathrm{BrdU}$ in the nucleus but not in the micronucleus. The number of cells showing no BrdU in the nucleus or in the micronucleus increased after DES but not after 5-azacytidine treatment. In fact, 5azacytidine decreased the number of cells in this category.

Effects of 5-azacytidine on the mitotic apparatus. Because of reports that 5-azacytidine is an aneugen (Van Hummelen et al., 1992) and our data showing that some of the micronuclei could contain whole chromosomes, we decided to examine whether mechanisms known to induce aneuploidy are operable in the response of L5178Y cells to this compound. Many aneuploidy inducing agents affect the metaphase chromosome ring arrangement and spindle morphology. Consequently, we compared the effects of 5-azacytidine with DES, an aneuploidy inducing agent, on the mitotic ring arrangement (Table 3) and on spindle morphology by anti-tubulin antibody staining. As controls, we used untreated cells. Out of more than 2000 evaluated rings, only one showed a displaced chromosome and spindle morphology looked normal after 5-azacytidine treatment. This was comparable to vehicle controls which did not have any displaced chromosomes. The DES-

\footnotetext{
bisbenzimid staining. (A) Anaphase chromatid separation. (B) Telophase with chromatid bridge.
} 
treated cells showed distorted spindles along with an elevated number of rings with displaced chromosomes (12 of 264 rings) from the metaphase plate. The DES-induced disturbance of the metaphase ring arrangement occurred during the 4-h treatment and, therefore, was an effect on cells that were in $G_{2}$ or mitosis. To exclude the possibility that 5 -azacytidine might only have an effect on the metaphase ring arrangement after it has been integrated into the DNA in S-phase, we investigated different time points up to $5 \mathrm{~h}$ after the 4-h treatment. However, at none of the observation times did 5-azacytidine have any detectable effect on the formation of the metaphase plate.

To investigate 5-azacytidine treated cells during the course of mitosis, we used supravital UV microscopy to visualize the distribution of chromatin elements throughout mitosis. L5178Y cells grow in suspension and do not attach to the observation chamber. Therefore, SHE cells were used for this purpose. We observed that the development of the metaphase plate from prophase to metaphase was normal in 5-azacytidine-treated cells. This is consistent with the metaphase ring arrangement results and indicates that there were no immediate, visible effects due to 5-azacytidine treatment. However, the cells showed impaired chromatid disjunction at the onset of anaphase which resulted in chromatid bridges which sometimes were sustained into interphase (Fig. 5). In 20 control mitoses, the one chromatid bridge that still existed in ana-telophase (5\%) developed a normal interphase. We followed 16 mitoses in the first $4 \mathrm{~h}$ after 5 -azacytidine addition. Of these, the one cell $(\sim 6 \%)$ showing an ana-telophase chromatid bridge developed a normal interphase. At later observation times (4-30 h after 5azacytidine addition), 7 of $33(\sim 21 \%)$ mitoses followed showed ana-telophase bridges; 2 of these $(\sim 6 \%)$ persisted until interphase. Thus, since all the mitoses that were followed during the first 4 $\mathrm{h}$ after 5-azacytidine addition seemed normal, we hypothesized that 5-azacytidine had to be integrated into the DNA during $S$ phase for its effect on mitosis to appear in late metaphase.

We also noted that, in some cases, the chromosomes in cells treated with 5-azacytidine were not able to separate during mitosis (endomitosis).
This occurred once in 20 control mitoses, once in 16 mitoses followed at $20-30 \mathrm{~h}$ after 5-azacytidine addition, and in 11 of 17 mitoses $(65 \%)$ between 4 and $10 \mathrm{~h}$ after 5-azacytidine addition. This last effect occurred during the first mitosis after 5-azacytidine treatment.

\section{Discussion}

In this paper we demonstrate that 5-azacytidine induces micronuclei in L5178Y cells at concentrations at which we and others have previously reported its mutagenic activity at the $t k$ locus (Amacher and Turner, 1987; McGregor et al., 1989). The results of the CREST, c-banding and hybridization experiments suggest that the induced micronuclei contained mostly chromosomal fragments, although the presence of some whole chromosomes is possible.

Our results are consistent with other reports of the compound's clastogenicity (Call et al., 1986; Shelby, 1992; Stopper et al., 1992; Fucik, 1970; Walker et al., 1987). However, Van Hummelen and coworkers (Van Hummelen et al., 1992) asserted that 5-azacytidine is an aneugen. These investigators reported that this compound induced micronuclei in mouse bone-marrow cells in vivo and that the micronuclei contained whole chromosomes. They found that approximately $80 \%$ of the micronuclei induced by 5 -azacytidine in mouse bone marrow are centromere-positive. In their paper they argue that CREST antibody staining results can be artifactual, especially with a compound like 5-azacytidine because, by changing the methylation status of the centromere, the chemical probably inactivates kinetochore proteins resulting in kinetochore-negative micronuclei that contain whole chromosomes. This hypothesis is not supported by our data, in which the CREST antibody staining and the centromeric heterochromatin DNA-staining results are similar suggesting that only a small percentage of micronuclei could contain whole chromosomes with inactivated kinetochores. These results support the conclusion that 5-azacytidine-induced micronuclei contain mostly chromosome fragments.

Our data demonstrate that 5-azacytidine did not induce micronuclei via interference with 
chromosome distribution during prophase or metaphase or by lack of kinetochore binding, further ruling out the possibility that 5 -azacytidine has predominantly aneugenic activity. There was no effect on metaphase ring arrangements and spindle morphology. On the other hand, our data do demonstrate that visible effects of 5azacytidine could be observed after the cells entered anaphase. Chromatid bridges were observed in anaphase that sustained until telophase and even in some cases until interphase. In the first mitosis after 5-azacytidine treatment and removal, we observed an increase in the rate of endomitosis. At this first mitosis, the methylation patterns at individual $\mathrm{CpG}$ sequences on the two DNA strands would be different, some $\mathrm{CpG}$ sequences being methylated on both strands and others hemimethylated (methylated on one strand) due to 5-azacytidine incorporation. In subsequent mitoses, these $\mathrm{CpG}$ sequences of both strands would be methylated in $3 / 4$ of the progeny cells; the remaining $1 / 4$ would be hypomethylated (contain unmethylated $\mathrm{CpG}$ sequences on both strands). Thus, only in the first mitosis could a hemimethylated state have existed. It appears that maximum endomitosis occurred during mitosis of cells with hemimethylated DNA. It has been shown that, in addition to endoreduplication, maximal SCE induction also occurred during the first mitosis after treatment (Hori, 1983).

Although the effects of 5-azacytidine were not visible in supravital UV microscopy until anaphase and telophase, our BrdU incorporation studies showed that the cells had to be in $S$ phase at the time of exposure for micronuclei to be formed.

There are two internally consistent hypotheses that can explain the BrdU incorporation data. In the first we assume that 5-azacytidine can induce DNA repair in the nucleus, but not in the micronucleus and that this repair response is activated through a mechanism that does not involve incorporation of 5-azacytidine into DNA and, therefore, operates outside of $S$ phase. Whether 5 -azacytidine can induce repair by its incorporation into DNA is not relevant to this scenario because such an effect would show the presence of $\mathrm{BrdU}$ in both the micronuclei and nuclei and could not be distinguished from normal replica- tion. In this scenario, the increase in cells treated with 5-azacytidine showing BrdU in both the nuclei and micronuclei would be due to incorporation during $S$ phase replication. An increase of cells in the second category where BrdU is incorporated into the nucleus but not the micronucleus would be due to repair. In the final category in which BrdU was not incorporated into the cell at all, 5-azacytidine treatment decreased the number of cells with no BrdU in either the nucleus or micronucleus. Thus, the loss of micronuclei with no BrdU would have to be due to a shift of some of the cells that would have appeared in the third category if treated only with the vehicle control to the second category in which the nucleus, but not the micronucleus, contains BrdU due to repair.

Based on our understanding of how 5-azacytidine interacts with cells, the assumption that 5azacytidine can induce repair when applied to cells during non-replicative phases of the cell cycle is difficult to accept since 5-azacytidine is not known to bind to DNA. There is no evidence that 5 -azacytidine induces repair; in fact a report examining the effects of this compound on unscheduled DNA synthesis (UDS) in SHE cells was negative (Stopper et al., 1992). However, it is thought that UDS is sensitive only to large patch repair. Whether the assumptions made are valid or not, this scenario asserts that SHE cells must be in $S$ phase for micronuclei to be induced by 5-azacytidine.

In the second mechanism we assume that 5azacytidine does not induce repair and that BrdU incorporation into nuclear DNA implies that the cells were exposed in $S$ phase. In this scenario, 5-azacytidine induces micronuclei when incorporated into DNA during replication as evidenced by the increase observed in the first category (BrdU incorporation into nuclear and micronuclear DNA). To explain the effects seen in the next two categories, we invoke an observation by Poot (Poot et al., 1990) that 5-azacytidine induces, among other cell cycle disturbances, a shortening of $G_{1}$. Any cells with pre-existing micronuclei that would have been in $G_{1}$ if not treated with 5-azacytidine, but now are in early $S$ phase because of the treatment, may have incorporated BrdU into the nuclear, but not the mi- 
cronuclear DNA. This would explain both the increase in 5-azacytidine-induced micronuclei in the second category and the reduction of micronuclei in 5-azacytidine-treated cells compared to the control in the third category. In this scenario, 5-azacytidine would simply induce a shift in micronuclei containing cells in category 3 to category 2.

Whichever of these scenarios is correct, it should be noted that both postulate that the exposure of SHE cells to 5-azacytidine in S phase will result in micronucleus induction. This is the main conclusion to be drawn from this experiment.

In contrast, DES, which induced micronuclei containing whole chromosomes, showed no increase in BrdU positive micronuclei when the cells were exposed in $S$ phase (category 1). However, an increase in BrdU-negative micronuclei in the presence of nuclear incorporation of $\mathrm{BrdU}$ was registered. This may suggest that DES changes the cell cycle through mechanisms similar to that proposed for 5-azacytidine. The number of cells showing no BrdU signal in the nucleus and the micronucleus increased after DES treatment. These cells could have been in $G_{2}$ phase during treatment and formed micronuclei during the mitoses immediately following treatment or they could have been in $\mathrm{M}$ phase, in which case the micronuclei would have been formed in the same mitosis in which treatment occurred. Our data for DES are in agreement with the published ideas about that compound's mechanism of micronucleus induction (Schiffmann and DeBoni, 1991).

Our observation of a delay between 5-azacytidine treatment and the occurrence of mitotic damage and our conclusion that 5-azacytidine had to be integrated into DNA in S-phase to manifest its effects in mitosis is consistent with reports that 5-azacytidine, when added to cells during late $S$ phase, prevents the condensation of R-band positive heterochromatin (ViegasPequignot and Dutrillaux, 1981). Hori also reported that pulse treatment of $\mathrm{CHO}$ cells and kangaroo-rat cells during $S$ phase resulted in segmented metaphase chromosome decondensation (Hori, 1983). The most sensitive sites, in that study, appeared to be heterochromatic regions of chromosomes which replicated late in S phase.

The observation that the effects of 5-azacytidine are not produced until late metaphase is also supported by our observation that 5-azacytidine had no effect on spindle morphology and that the metaphase plate looked normal. These results contrasted with those obtained with DES which showed effects on spindle morphology and the metaphase plate. Albertini (Albertini et al., 1988) has also reported that spindle tubulin assembly is not influenced by 5-azacytidine treatment.

Thus, all our data taken together indicate that 5 -azacytidine is predominantly a clastogen. Although it is possible that 5-azacytidine has some aneugenic properties, we could not establish the existence of any such mechanism.

5-Azacytidine is often used to induce hypomethylation in cells. The biological consequences resulting from this treatment are usually attributed to this change in methylation which is, in turn, generally assumed to interfere with the transcriptional machinery directly. However, the chemical has other biochemical effects that could also have biological consequences and often these are ignored. Given what is known about the effects of 5-azacytidine on the DNA structure and its ability to induce chromosomal aberrations when incorporated into DNA during $S$ phase, we have to consider that the mechanism leading to transcriptional changes are more complicated than simply a change in methylation. Methylated regions can undergo transitions from the $B$ to the $\mathrm{Z}$ form of DNA at physiological conditions, whereas, if these regions were unmethylated, they would exist only in the $B$ form (Banerjee and Benedict, 1979; Nickol et al., 1982). DNA stretches containing unmethylated $\mathrm{GC}$ regions are less stable than those that are methylated (Collins and Myers, 1987). Methylated CpG rich regions of DNA are more resistant to micrococcal nucle. ase digestion than bulk DNA (Keshet et al., 1986: Razin and Cedar, 1977; Solage and Cedar, 1978). In an added layer of complication, MspI, which is able to cut CCGG sequences in naked DNA whether or not the internal $C$ is methylated, is unable to cleave methylated $\mathrm{CpG}$ in nuclei suggesting that DNA binding proteins may have different affinities to methylated and unmethylated 
sites (Antequera et al., 1989, 1990; Meehan et al., 1989). $\mathrm{CpG}$ rich heterochromatic sites are targets for 5-azacytidine induced demethylation and also for decondensation due to a more open conformation. This may make those stretches more accessible to repair enzymes resulting in regions that are more susceptible to the production of chromosomal aberrations induced by these enzymes (Savage, 1975). Our results with ethionine, which is considered to be a hypomethylating agent, supports this idea. As observed by others, GC rich regions are particularly sensitive to the decondensation effects of 5-azacytidine (Fucik et al., 1970; Hori, 1983; Parrow et al., 1989; Schmid et al., 1984). The decondensation or out of cycle condensation effects may be responsible for the formation of the chromatid bridges (incomplete untangling of the chromatids) before separation in anaphase. These chromatid bridges may be caused by the same mechanism that results in the endomitosis that we and others have observed (Fucik et al., 1970; Hori, 1983; Stopper et al., 1992). As an example, DNA topoisomerase II, an enzyme with an essential role in chromatid separation, may be impaired in its function when interacting with hemi- or hypo-methylated or undercondensed DNA. This has been suggested for minor groove binding agents like berenil which may cause inhibition of topoisomerase II due to their effects on chromosome conformation (Poot et al., 1990) and is supported by our findings with berenil. We could envision a mechanism whereby the formation of chromatid bridges results in breakage of chromatids and the chromatin fragments are enclosed in micronuclei. The existence of such a mechanism is supported by our observation that $80 \%$ of the micronuclei did not contain whole chromosomes, although we did not find chromatin fragments lying apart from the metaphase ring arrangement. This can only be explained if chromosome breakage occurred after the formation of the metaphase plate. However, the experiments of Davidson and coworkers (Davidson et al., 1992) seem to contradict this mechanism. Using phase contrast video microscopy, they also observed that 5-azacytidinetreated cells were unable to separate in the first cell division after treatment. This was probably due to endomitosis. They also observed that cel- lular bridges were formed. Nevertheless, they concluded that these bridges did not break. Therefore, we cannot rule out other mechanisms of 5-azacytidine mediated micronucleus induction, such as elimination of improperly condensed chromatin material via micronucleus formation during the interphase of the cell cycle.

\section{Acknowledgements}

We wish to acknowledge the support of the Deutsche Forschungsgemeinschaft, Sonderforschungsbereich 172 and also of the Hauptverband der Gewerblichen Berufsgenossenschaften, W5205 St. Augustin, Germany. We wish to thank Drs. Michael Shelby, Jack Bishop and Graham Hook for their comments on this manuscript.

\section{References}

Albertini, S., U. Friederich, C. Holderegger and F.E. Würgler (1988) The in vitro porcine brain tubulin assembly: effects of a genotoxic carcinogen (aflatoxin $B_{1}$ ), eight tumor promoters and nine miscellaneous substances, Mutation Res., 201, 282-292.

Amacher, D., and G. Turner (1987) The mutagenicity of 5-azacytidine and other inhibitors of replication DNA synthesis in the L5178Y mouse lymphoma cell, Mutation Res., 176, 123-131.

Antequera, F., D. Macleod and A.P. Bird (1989) Specific protection of methylated CpGs in mammalian nuclei, Cell, 58, 509-517.

Antequera, F., J. Boyes and A. Bird (1990) High levels of de novo methylation and altered chromatin structure at $\mathrm{CPG}$ islands in cell lines, Cell, 62, 503-514.

Banerjee, A., and W. Benedict (1979) Production of sister chromatid exchanges by various cancer chemotherapeutic agents, Cancer Res., 39, 797-799.

Call, K., J. Jensen, H. Liber and W. Thilly (1986) Studies of mutagenicity and clastogenicity of 5-azacytidine in human lymphoblasts and Salmonella typhimurium, Mutation Res., $160,249-257$.

Clive, D., W.G. Flamm, M.R. Machesko and N.J. Bernheim (1972) Mutational assay system using the thymidine kinase locus in mouse lymphoma cells, Mutation Res., 16, 77-87.

Collins, M., and R.M. Myers (1987) Alterations in DNA helix stability due to base modification can be evaluated using denaturing gradient gel electrophoresis, J. Mol. Biol., 198, 737-744.

Constantinides, P.G., P.A. Jones and W. Gevers (1977) Functional striated muscle cells from non-myoblast precursors following 5-azacytidine treatment, Nature(London), 267, 364-366.

Daston, D.L., Z. Kelecsényi, R.A. Whitaker, K.C. Hines, W.J. 
Caspary and K.R. Tindall (1992) 5-Azacytidine mutagenesis in AS52 cells, Environ. Mol. Mutagen., 19 (Suppl. 20), 13.

Davidson, S., P. Crowther, J. Radley and D. Woodcock (1992) Cytotoxicity of 5-aza-2'-deoxycytidine in a mammalian cell system, Eur. J. Cancer, 28, 362-368.

Degrassi, V.L., and C. Tanzarella (1988) Immunofluorescent staining of kinetochores in micronuclei: A new assay for the detection of aneuploidy, Mutation Res., 167, 339-345.

Fucik, V., A. Michaelis and R. Rieger (1970) On the induction of segment extension and chromatid structural changes in Vicia faba chromosomes after treatment with 5-azacytidine and 5-azadeoxycytidine, Mutation Res., 9, 599-606.

Holliday, R. (1989) A different kind of inheritance, Sci. Am., 60-73.

Hori, T.A. (1983) Induction of chromosome decondensation, sister-chromatid exchanges and endoreduplications by 5 azacytidine, an inhibitor of DNA methylation, Mutation Res., 121, 47-52.

Jones, P.A., and S.M. Taylor (1980) Cellular differentiation, cytidine analogs and DNA methylation, Cell, 20, 85-93.

Kelecsényi, Z., D.L. Spencer, W.J. Caspary and K.R. Tindall (1993) Molecular analyses of 5-azacytidine induced mutations in AS52 cells. Environ. Mol. Mutagen., in press.

Keshet, I., J. Lieman-Hurwitz and H. Cedar (1986) DNA methylation affects the formation of active chromatin, Cell, 44, 535-545.

Kirchner, S., H. Stopper, T. Papp, I. Eckert, H.J. Yoo, B.K. Vig and D. Schiffmann (1992) Cytogenetic changes in primary, immortalized and malignant mammalian cells, Toxicol. Lett., in press.

Lavia, P., M. Ferraro, A. Micheli and G. Olivieri (1985) Effect of 5-azacytidine (5-aza C) on the induction of chromatid aberrations (CA) and sister-chromatid exchanges (SCE), Mutation Res., 149, 463-467.

McGregor, D.B., A.G. Brown, P. Cattanach, W. Shepherd, C. Riach, D.S. Daston and W.J. Caspary (1989) TFT and 6TG resistance of mouse lymphoma cells to analogs of azacytidine, Carcinogenesis, 10, 2003-2008.

Meehan, R.R., J.D. Lewis, S. McKay, E.L. Kleiner and A.P. Bird (1989) Identification of mammalian protein that binds specifically to DNA containing methylated CpGs, Cell, 58, 499-507.

Nickol, J., M. Behe and G. Felsenfeld (1982) Effect of the B-Z transition in poly $\left(\mathrm{dG}-\mathrm{m}^{5} \mathrm{dC}\right)$ poly $\left(\mathrm{dG}-\mathrm{m}^{5} \mathrm{dC}\right)$ on nucleosome formation, Proc. Natl. Acad. Sci. (U.S.A.), 79, 1771-1775.

Parrow, V.C., P. Alestrom and K.M. Gautvick (1989) 5Azacytidine induced alterations in $\mathrm{GH} 12 \mathrm{Cl}$ cells: effect on cellular morphology, chromatin structure, DNA and protein synthesis, J. Cell. Sci., 93, 533-543.

Poot, M., J. Koehler, P.S. Rabinovitch, H. Hoehn and J.H.
Priest (1990) Cell kinetic disturbances induced by treatment of human diploid fibroblasts with 5-azacytidine indicate a major role for DNA methylation in the regulation of the chromosome cycle, Hum. Genet., 84, 258-262.

Razin, A., and H. Cedar (1977) Proc. Natl. Acad. Sci. (U.S.A.), $75,2725-2728$.

Savage, J.R.K. (1975) Classification and relationships of induced chromosomal structural changes, J. Med. Genet., $12,103-122$.

Schiffmann, D., and U. DeBoni (1991) Dislocation of chromatin elements in prophase induced by diethylstilbestrol: a novel mechanism by which micronuclei can arise, Mutation Res., 246, 113-122.

Schmid, M., T. Haaf and D. Grunert (1984) 5-Azacytidine-induced undercondensations in human chromosomes, Hum. Genet., 67, 257-263.

Schmuck, G., R. Pechan, D. Wild, D. Schiffmann and D. Henschler (1988) SOS-dependent mutagenic activity of 5-azacytidine in Salmonella, Mutation Res., 175, 205-208.

Shelby, M. (1992) Chromosomal aberrations in CHO cells, unpublished results.

Solage, A., and H. Cedar (1978) Biochemistry, 17, 2934-2938.

Stopper, H., R. Pechan and D. Schiffmann (1992) 5-Azacytidine induces micronuclei in and morphological transformation of Syrian hamster embryo ffbroblasts in the absence of unscheduled DNA synthesis, Mutation Res., 283, 21-28.

Sumner, A.T. (1972) A simple technique for demonstrating centromeric heterochromatin, Exp. Cell. Res., 75, 304-306.

Taylor, S.M., and P.A. Jones (1979) Multiple new phenotypes induced in 10T1/2 and 3T3 cells treated with 5-azacytidine, Cell, 17, 771-779.

Van Hummelen, P., A. Deleener, P. Vanparys and $\mathbf{M}$. Kirsch-Volders (1992) Discrimination of aneuploidogens from clastogens by c-banding, DNA and area measurements of micronuclei from mouse bone marrow, Mutation Res., 271, 13-28.

Viegas-Pequignot, E., and B. Dutrillaux (1981) Detection of G-C rich heterochromatin by 5-azacytidine in mammals, Hum. Genet., 57, 134-137.

Von Hoff, D., M. Slavik and F. Muggia (1976) 5-Azacytidine: A new anticancer drug with effectiveness in acute myelogenous leukemia, Annu. Int. Med., 85, 237-245.

Walker, C., A. Matthews and J. Shay (1987) Suppression of tumorigenicity mediated by 5 -azacytidine and associated with increased chromosome number, J. Natl. Cancer Inst., $78,695-700$.

Zatsepina, O.V., V.O. Airapetian and G.I. Kir'ianov (1989) The effect of 5-azacytidine on the cell cycle and chromosome structure in cell cultures of swine embryonal kidney tissue, Mol. Biol. Mosk., 23, 772-782. 